\title{
LncRNA XIST knockdown alleviates LPS-induced acute lung injury by inactivation of XIST/miR-132-3p/MAPK14 pathway
}

\author{
XIST promotes ALI via miR-132-3p/MAPK14 axis
}

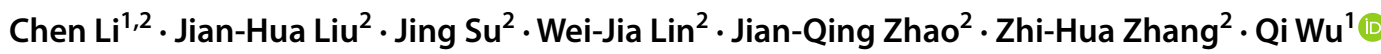 \\ Received: 20 May 2021 / Accepted: 20 July 2021 / Published online: 3 August 2021 \\ (c) The Author(s), under exclusive licence to Springer Science+Business Media, LLC, part of Springer Nature 2021
}

\begin{abstract}
Acute lung injury (ALI) is a fatal inflammatory response syndrome. LncRNA XIST (XIST) is a lung cancer-related gene and participates in pneumonia. However, whether XIST participates in lipopolysaccharides (LPS)-induced ALI remains unclear. LPS-induced inflammation model was constructed in vitro, then cell viability, cytokines, cell apoptosis, protein, and mRNA expressions were individually detected by cell counting kit-8, enzyme-linked immunosorbent assay and flow cytometry, Western blot, and qRT-PCR. A dual-luciferase reporter assay confirmed the relationships among XIST, miR-132-3p, and MAPK14. Furthermore, inflammation and conditions after knockdown of XIST were assessed by hematoxylin and eosin staining, lung wet-to-dry weight ratio, $\mathrm{PaO}_{2} / \mathrm{FiO}_{2}$ ratio, and malondialdehyde (MDA) contents using LPS-induced in vivo model. Our findings indicated that the LPS challenge decreased cell viability, increased cell apoptosis, and caused secretions of pro-inflammatory cytokines. Noticeably, LPS significantly upregulated XIST, MAPK14, and downregulated miR-132-3p. Mechanistically, XIST acted as a molecular sponge to suppress miR-132-3p, and MAPK14 was identified as a target of miR132-3p. Functional analyses demonstrated that XIST silencing remarkably increased cell survival and alleviated cell death and lung injury through decreasing TNF- $\alpha$, IL-1 $\beta$, IL-6, accumulation of inflammatory cells, alveolar hemorrhage, MDA release, and increased $\mathrm{PaO}_{2} / \mathrm{FiO}_{2}$ ratio, as well as upregulating Bcl-2, and downregulating Bax, MAPK14, and p-extracellular signal-regulated kinases $1 / 2$. In contrast, inhibition of the miR-132-3p antagonized the effects of XIST silencing. In conclusion, inhibition of XIST exhibited a protective role in LPS-induced ALI through modulating the miR-132-3p/MAPK14 axis.
\end{abstract}

Keywords XIST $\cdot \operatorname{miR}-132-3 p \cdot M A P K 14 \cdot$ Acute lung injury $\cdot$ LPS

\section{Abbreviations}

LncRNAs Long Noncoding RNAs

XIST X-Inactive Specific Transcript

miRNAs MicroRNAs

ALI Acute Lung Injury

MAPK14 Mitogen-Activated Protein Kinase 14

ERK1/2 Extracellular Signal-Regulated Kinases 1/2

\section{Qi Wu}

qqwwwui1223@163.com

1 Department of Respiratory and Critical Care Medicine,

Tianjin Medical University General Hospital, No.

154, Anshan Road, Heping District, Tianjin 300052,

People's Republic of China

2 Department of Respiratory and Critical Care Medicine, The First Affiliated Hospital of Hebei North University, Zhangjiakou 075000, Hebei Province, People's Republic of China

$\begin{array}{ll}\text { LPS } & \text { Lipopolysaccharides } \\ \text { LBP } & \text { Lipopolysaccharide Binding Protein } \\ \text { ELISA } & \text { Enzyme-Linked Immunosorbent Assay } \\ \text { ARDS } & \text { Acute Respiratory Distress Syndrome } \\ \text { 3'-UTRs } & \text { 3'-Untranslated Regions } \\ \text { CCK-8 } & \text { Cell Counting Kit-8 } \\ \text { H\&E } & \text { Hematoxylin and Eosin } \\ \text { qRT-PCR } & \text { Quantitative Real-Time Polymerase Chain } \\ & \text { Reaction }\end{array}$

\section{Introduction}

Acute lung injury (ALI) is an urgent respiratory dysfunction characterized by pulmonary infiltration, acute severe hypoxia, and edema. Both ALI and its related acute respiratory distress syndrome (ARDS) are devastating clinical 
conditions that lead to high mortality rates at $30-40 \%$ [1]. ALI is a multifactorial disease directly related to some conditions such as pneumonia, pulmonary contusion, post lung transplantation, or closely related to some situations such as sepsis and multiple trauma [2]. Lipopolysaccharide (LPS) is an endotoxin derived from the outer membrane of Gramnegative bacteria. It can bind to the extracellular binding proteins lipopolysaccharide-binding protein (LBP), CD14, and MD-2, then further interacts with toll-like receptor 4, and triggers inflammation by activating extracellular signal-regulated kinases (ERK) $1 / 2$ and p38 mitogen-activated protein kinase (p38 MAPK) pathways. After activation of these kinases, the expression of pro-inflammatory genes is significantly enhanced. Meanwhile, the LPS challenge also enhanced neutrophil infiltration into the lung [3]. Hence, LPS is a critical factor in the pathophysiology of ALI, but the definite mechanisms remain elusive. Up to date, there is still a lack of effective specific drug therapy for ALI. Therefore, acute inflammation is a good topic responsible for ALI.

Human Genome Project had confirmed that only a tiny portion of the mammalian genome is transcribed into protein-coding RNA, and about $80 \%$ is noncoding RNAs [4]. Long noncoding RNAs (LncRNAs) comprise more than 200 nucleotides that do not encode proteins. LncRNAs can fold into complex structures and interact with proteins, DNA, and other RNA and participate in many genetic modifications [5]. LncRNA X-inactive specific transcript (XIST) is a noncoding RNA on the X-chromosome of placental mammals. It contains two long stem-loop structures that each comprised four repeats. As the master regulator, XIST plays a critical role in X-chromosome inactivation[6]. Previous literature pointed out that XIST performed biological activities via acting as macromolecular scaffolding for protein recruitment to adopt discrete 3D-folding, as molecular sponges for sequestering regulatory ncRNAs or proteins, and as a genomic 3D organizer cis/trans-regulatory elements regulating transcription and RNA splicing [7]. XIST is transcribed from the future inactive $\mathrm{X}$-chromosome. Functional analysis revealed that XIST could interact directly with PRC complex PRC2 or SHARP to silence genes [8]. Recently, several studies showed that dysregulations of XIST played vital roles in many diseases, such as non-small-cell lung cancer $[9,10]$, coronary artery disease [11], Parkinson's disease [12], Alzheimer's diseases [13], and Hodgkin's disease [14]. Recently, the increasing evidence showed that XIST was a critical regulator during the pathological progress of acute injuries, such as acute pneumonia [15], acute kidney injury [16], myocardial injury [17], and liver injury [18]. However, the exact roles of XIST in ALI still were largely unknown.

MicroRNAs (miRNAs) contain about 18-24 nucleotides. Like LncRNAs, miRNAs also can bind to mRNA 3 '-untranslated regions (3'-UTRs) to promote their degradation or inhibit their translation. Importantly, miR-132-3p could activate multiple signaling pathways and is involved in many diseases such as tumorigenesis [19], myocardial injury [20], and brain ischemic injury [21]. Recently, some experiments showed that miRNAs such as miR-34b-5p [22], miR-33 [23], miR-223 [24], and miR-128-3p [25], as well as miR-132-3p [26] could regulate the process of the inflammatory process. However, there is still no report about how miR-132-3p participates in the pathophysiology of ALI and what molecular pathogenesis mechanisms are involved.

In mammals, the family of MAPKs is composed of ERK, p38, and c-Jun NH2-terminal kinase (JNK). Each MAPK signaling axis consists of at least three components: a MAPKKK kinase (MAP3K), a MAPKK kinase (MAP2K), and a MAPK. MAPKs could actively participate in cell survival, apoptosis, and differentiation [27]. In humans, p38 MAPKs encoded by the MAPK14 gene is ubiquitously expressed in many cell types. p38 MAPKs signaling pathway is essential for the modulation of neutrophil activation, especially for endotoxin stimulus. Hence, MAPK14 might play a key role in lung inflammatory response. However, we still did not know how MAPK14 regulates the pathological process of ALI.

Here, we investigated the expressions of XIST and miR132-3p in LPS-induced ALI models and confirmed XIST as a ceRNA that directly targeted the miR-132-3p and miR$132-3 p$ directly interacted with MAPK14 by binding to its 3'-UTR. Further experiments showed that knockdown of XIST might alleviate LPS-induced ALI by modulating the miR-132-3p/MAPK14 pathway, which provided us new evidence to treat ALI.

\section{Materials and methods}

\section{Cell culture}

MLE-12 cells (mouse lung epithelial cells) were purchased from the American Type Culture Collection (CRL-2110, Manassas, VA, USA) and cultured in HITES medium supplemented with $2 \%$ fetal bovine serum (ATCC, 30-2020). Cells were maintained in a humidified $5 \% \mathrm{CO}_{2}$ at $37{ }^{\circ} \mathrm{C}$. Subsequent experiments were implemented when cells reached 80\% confluence. LPS (L4391) was bought from Sigma (St. Louis, MO, USA). For ALI in vitro cell model, LPS $(1 \mu \mathrm{g} / \mathrm{mL})$ was used to treat MEL-12 for $24 \mathrm{~h}$.

\section{Plasmid constructions and cell transfection}

Wide typed or mutant pcDNA3.1 targeting XIST overexpression vector (XIST-wt, XIST-mut), MAPK14 overexpression vector (MAPK14-wt, MAPK14-mut), and negative control (NC) were all designed, amplified, and subcloned into pcDNA3.1 vector by Genepharma (Shanghai, China). 
si-XIST, siRNA negative control (si-NC), mimic negative control (mimic NC), miR-132-3p mimic, inhibitor negative control (inhibitor NC), miR-132-3p inhibitor were purchased by Genepharma (Shanghai, China). The lentivirus vectors to inhibit XIST was bought by Genepharma (Shanghai, China). The lentiviruses were packaged in HEK293T cells, which were named lenti-si-XIST and lenti-si-NC, respectively. Lipofectamine 2000 (Invitrogen, USA) was applied for cell transfection following the manufacturer's instructions.

\section{Cell counting kit (CCK)-8}

MLE-12 cells were seeded into 96-well plates. After $24 \mathrm{~h}$, $1 \mu \mathrm{g} / \mathrm{mL}$ LPS was added to fresh medium for further $24 \mathrm{~h}$ incubation, then cell viability was detected. For the assessment of the role of XIST, MLE-12 cells were transfected with si-NC or si-XIST and stimulated by LPS $(1 \mu \mathrm{g} / \mathrm{mL})$. Subsequently, CCK-8 reagent (A311-01, Vazyme, Nanjing, China) was added to each well, followed by a further $2 \mathrm{~h}$ incubation. The OD values were detected at $450 \mathrm{~nm}$ using an ELISA plate reader (Thermo Fisher Scientific, Inc.). Individual experiment repeated three times.

\section{Enzyme-linked immunosorbent assay (ELISA)}

MLE-12 cells were transfected with si-NC and si-XIST, then treated by LPS $(1 \mu \mathrm{g} / \mathrm{mL})$ for $24 \mathrm{~h}$. The supernatant was collected to measure TNF- $\alpha$, IL- $1 \beta$, and IL- 6 using their corresponding ELISA kits TNF- $\alpha$, IL- $1 \beta$, and IL- 6 according to the instructions. All the ELISA kits were bought from Cusabio Technology LLC (Houston, TX, USA).

\section{Flow cytometry}

Cells after twice washes with cold $1 \times$ phosphate saline (PBS) were labeled with Annexin V/PI double-staining kit (BD Biosciences, MA, USA). The apoptotic cells were detected and analyzed by flow cytometry (FACSCalibur, BD, San Jose, CA).

\section{RNA extraction and quantitative real-time PCR (qRT-PCR)}

Total RNA and miRNA were extracted by RNeasy Mini Kit (Qiagen, Valencia, CA, USA) or mirVana miRNA isolation kit (Ambion, Austin, TX, USA). The concentrations and purity were analyzed by NanoDrop ND-2000 (Thermo Fisher, Wilmington, DE, USA). RNA was reversely transcribed by mRNA Reverse Transcription Kit (Thermo Fisher) or Taqman ${ }^{\mathrm{TM}}$ miRNA. The SYBR Premix Ex Taq (TaKaRa, Dalian, China) was used to assess XIST and MAPK14. $\beta$-actin was the internal control. miR-132-3p expression was detected using TaqMan MicroRNA Assay
(Applied Biosystems, Foster City, USA). U6 was the internal control. qRT-PCR was run on the Applied Biosystems 7500 Real-Time PCR System. The CT values were analyzed using the $2^{-\Delta \Delta C T}$ method. The primers used in this study were used: XIST sense AGG GTG TGT GTG CAT ATG GA and antisense CCG CCA TCT TTT CCT GTA CG; miR132-3p sense GGA CGG TAG CAA GCA AAG AGT GTG and antisense GGG ATT CTG GAA GAT GAT GAT GAC; MAPK14 sense TGA CCC TTA TGA CCA GTC CTT T and antisense GTC AGG CTC TTC CAC TCA TCT AT; $\beta$-actin sense GTG ACG TTG ACA TCC GTA AAG A and antisense GCC GGA CTC ATC GTA CTC C and U6 sense CTC GCT TCG GCA GCA CAT A and antisense AAC GAT TCA CGA ATT TGC GT.

\section{Western blot}

Total proteins were extracted using RIPA lysis buffer (Thermo Scientific ${ }^{\mathrm{TM}}$ ) supplemented by $0.1 \mathrm{mM}$ PMSF. After pre-cleared, protein concentrations were determined using a BCA protein assay kit (Pierce). Then, an equal amount of protein was applied to SDS-PAGE and then transferred to PVDF membranes (Millipore, Billerica, MA, USA). After blocking using $5 \%$ non-fat milk in $1 \times$ PBST, the primary antibodies were incubated overnight at $4{ }^{\circ} \mathrm{C}$. The secondary antibodies were incubated for $2 \mathrm{~h}$ at room temperature. The membranes were visualized using an ECL chemiluminescence kit (Fude, Hangzhou, China). The images were taken and analyzed by Image Lab 6.0. $\beta$-actin was the reference control. All the primary and secondary antibodies, including anti-Bcl-2 (sc-7382; 1:1000), anti-Bax (sc-7480; 1:1000), anti-MAPK14 (p38 $\alpha$ MAPK, sc-81621; 1:1000), anti-ERK1(sc-271269; 1:1000), anti-ERK2 (sc81457; 1:1000), anti-p-ERK1/2 antibody (Thr 202/Tyr 204) (sc-16982; 1:1000), anti- $\beta$-actin (sc-47778; 1:1000) and mouse anti-goat IgG-HRP secondary antibody (sc-2354, 1:11000) were purchased from Santa Cruz Biotechnology, Inc.

\section{Dual-luciferase reporter assay}

The potential binding sites among XIST, miR-132-3p, and MAPK14 were obtained from bioinformatics software StarBase V2.0. Site-Directed Mutagenesis Kit was used to mutate the potential binding sites in XIST and MAPK14 (SBS Genetech, Beijing, China). Then, the XIST or MAPK14 3'-UTR with the predicted binding site was amplified and subcloned into a pGL4.10 luciferase reporter vector (Promega, Madison, WI, USA) as XIST-wt, XISTmut, MAPK14-wt or MAPK14-mut, respectively. MEL-12 cells were co-transfected with miR-132-3p mimic/mimic NC and either XIST-wt/mut or MAPK14-wt/mut using Lipofectamine 2000 . The luciferase activity was determined after 
$48 \mathrm{~h}$ of post-transfection by a luciferase reporter assay kit (Promega, USA).

\section{Mice models of LPS-induced ALI}

40 male BALB/c mice were purchased from Liaoning Changsheng Technology Industrial Co., LTD (Certificate SCXK2010-0001; Liaoning, China). Mice were housed in a humidity-controlled environment with a light/dark cycle of $12 \mathrm{~h} / 12 \mathrm{~h}$ and fed with food and water ad libitum. This study was approved by the Ethics Committee of the Tianjin Medical University, and all experiments were performed following the National Institutes of Health guidelines. Mice were randomly divided into four groups $(n=10$ per group): Control group (saline), Model group ( $0.5 \mathrm{mg} /$ $\mathrm{kg}$ LPS, dissolved in saline), Model + si-NC group, and Model + si-XIST group. To induce in vivo ALI, LPS was administered to induce lung injury as previous reports with slight modifications [28]. Briefly, mice were anesthetized with pentobarbital sodium $(30 \mathrm{mg} / \mathrm{kg})$, followed by $10 \mu \mathrm{g}$ LPS in $50 \mu \mathrm{L}$ sterile saline with intratracheal injection (i.n.) with a 3-gauge needle. The same procedure gave the mice of the control group sterile saline. Then, the mice were placed in a vertical position and rotated for $1 \mathrm{~min}$ to distribute the installation in the lungs. For inhibition of XIST, mice had immediately received a tail vein injection of si-XIST (lentisi-XIST, $100 \mu \mathrm{L}$, virus titer $2 \times 10^{7}$ transduction unit [TU]/ $\mathrm{mL}$ ). The control mice received $100 \mu \mathrm{L}$ lenti-si-NC by tail vein injections. After $24 \mathrm{~h}$, mice were euthanized by $\mathrm{CO}_{2}$ asphyxiation. Subsequently, lung tissues were removed for other assays.

\section{Hematoxylin and eosin (H\&E) staining}

The left lungs were removed and fixed in $4 \%$ paraformaldehyde at $4{ }^{\circ} \mathrm{C}$ for $48 \mathrm{~h}$, then dehydrated by a series of graded ethanol and xylene, embedded and sectioned by cryostat (Leica CM1520). The sections were stained with H\&E and examined under a light microscope.

\section{Lung wet/dry (W/D) weight ratio}

Lung samples were collected $24 \mathrm{~h}$ after the LPS challenge. Each lung was blotted dry, weighed immediately as wet weight. Then the lungs were subjected to an oven at $80{ }^{\circ} \mathrm{C}$ for $72 \mathrm{~h}$ for the dry weight. The ratio of the wet lung weight to the dry lung weight was calculated to evaluate tissue edema.

\section{Assessment of $\mathrm{PaO}_{2} / \mathrm{FiO}_{2}$ ratio}

After $24 \mathrm{~h}$ administration by PBS or LPS with lenti-siXIST/lenti-si-NC, all the animals were given endotracheal intubation after anesthesia. Subsequently, pure oxygen $(7 \mathrm{~mL} / \mathrm{kg}$ ) was given later by mechanical ventilation. After ventilation for $15 \mathrm{~min}, \mathrm{PaO}_{2}$ analysis was measured on the blood samples obtained from the carotid artery using a GEM Premier 3000 gas analyzer (Instrumentation Laboratory, Italy). The oxygenation index was expressed as $\mathrm{PaO}_{2} / \mathrm{FiO}_{2}$.

\section{MDA assay}

The right lungs were collected and homogenized after the LPS challenge, and levels of MDA were determined using an MDA assay kit (A003-1, Jiancheng, Jiangsu, China). Procedures were according to the manufacturer's kit protocol.

\section{Statistical analysis}

All results are presented as means \pm SD. Data within different groups were compared using student's $t$-tests or one-way ANOVA analysis (ANOVA) using GraphPad Prism 7 (Graph Pad Software Co., San Diego, CA, USA). $P<0.05$ was considered statistically significant.

\section{Results}

\section{XIST, miR-132-3p, and MAPK14 were altered in LPS-induced MEL- 12 cells damage}

To explore the potential roles of XIST, miR-132-3p, and MAPK14, their expression levels were measured in LPSinduced MEL-12 cells. As shown in Fig. 1A, cell viability by CCK- 8 assay was significantly decreased after the LPS challenge. We found that the releases of TNF- $\alpha$, IL- $1 \beta$, and IL-6 were remarkably elevated (Fig. 1B-D). Cell apoptosis was also obviously increased after LPS treatment (Fig. 1E). By western blotting assay, it was shown that the level of Bcl-2 had notably decreased and Bax had increased after LPS stimulus (Fig. 1F). Meanwhile, we also found that MAPK14 and its related signaling molecules p-ERK1 and p-ERK2 were also highly upregulated (Fig. 1G). Additional experiments by qRT-PCR indicated that the LPS challenge increased XIST and decreased miR-132-3p (Fig. 1H and I). Taken together, XIST, miR-132-3p, and MAPK14 might be associated with the process of LPS-induced MEL-12 cell injuries.

\section{XIST knockdown promoted cell viability, alleviated LPS-induced injuries in MEL-12 cells}

To investigate the function of XIST during LPS-induced inflammatory injury, we transfected si-XIST to knockdown XIST in MEL-12, followed by LPS stimuli. qRT-PCR results showed that the XIST level was significantly inhibited after 
A

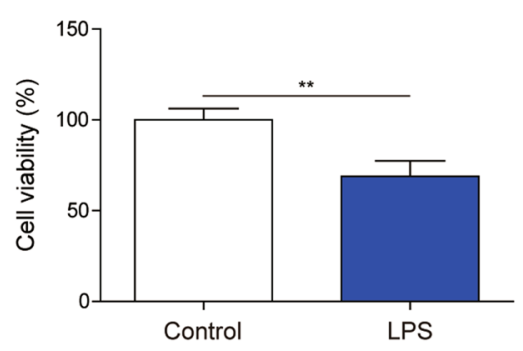

D

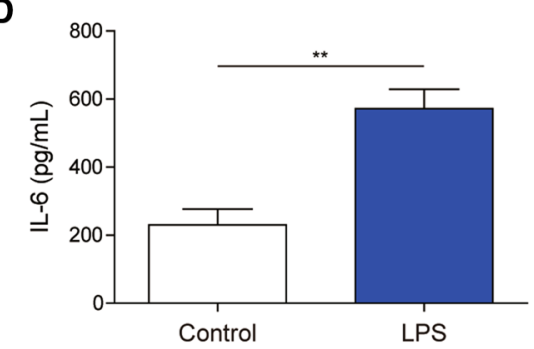

$\mathbf{F}$

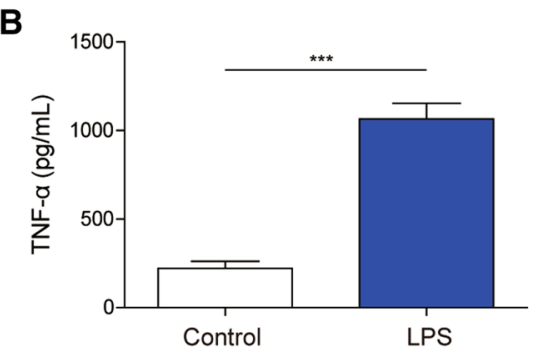

E

G
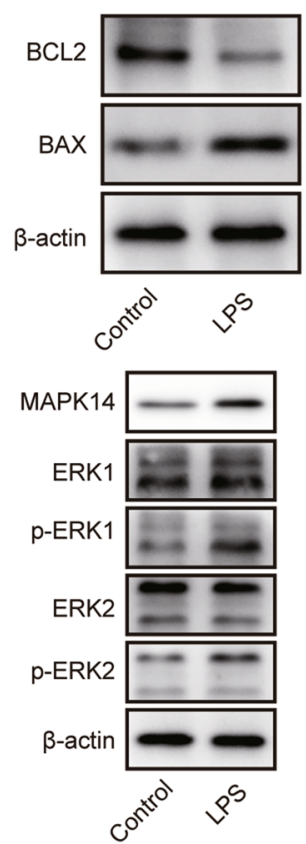
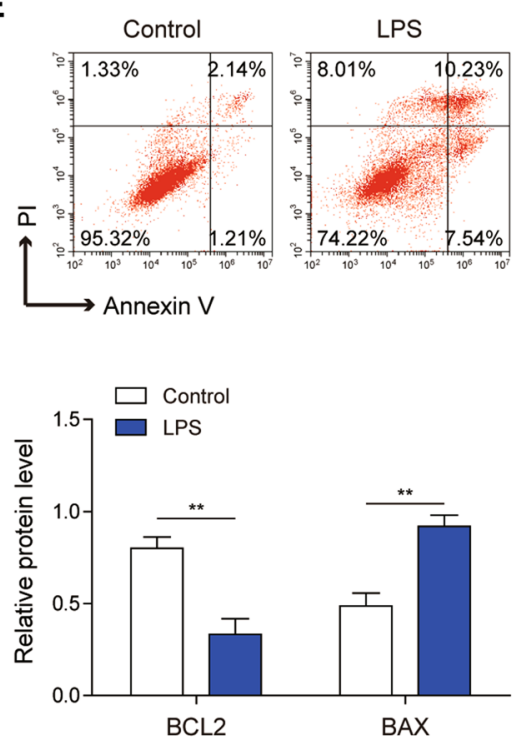

C
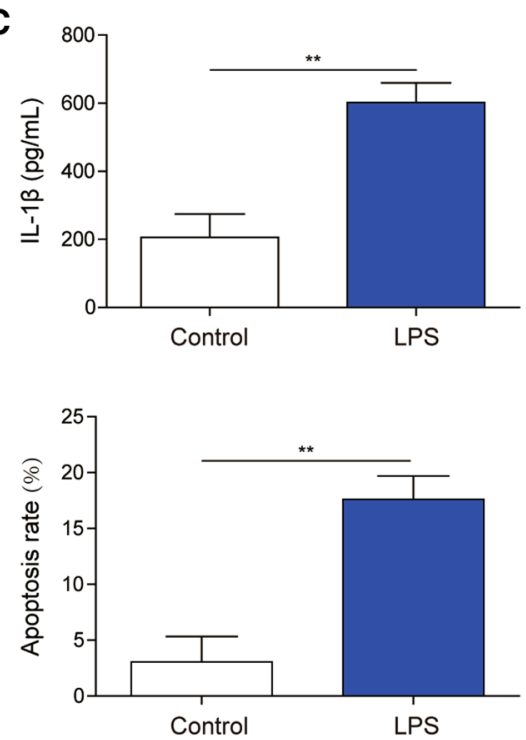

H

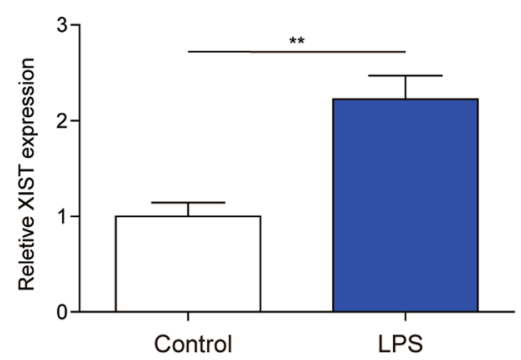

I

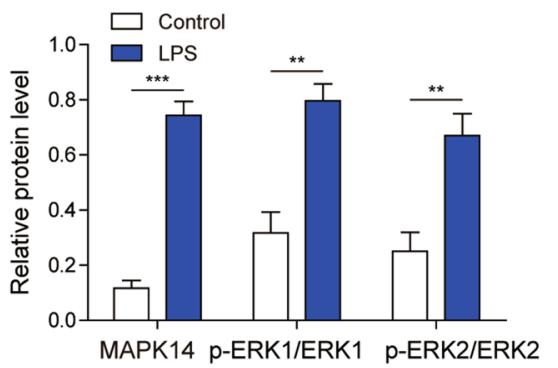

Fig. 1 XIST, miR-132-3p, and MAPK14 were altered in LPS-induced MEL-12 cells damage. A Cell viabilities were detected by CCK-8 assay. B-D ELISA assays measured TNF- $\alpha$, IL- $1 \beta$, and IL-6. E Cell

the knockdown of XIST (Fig. 2A). CCK-8 assay indicated knockdown of XIST alleviated LPS-induced cell death (Fig. 2B). Meanwhile, the releases of TNF- $\alpha$, IL-1 $\beta$, and IL-6 were attenuated due to XIST inhibition (Fig. 2C-E). Furthermore, LPS-induced cell apoptosis was also diminished because of the silence of the XIST gene (Fig. 2F). Also, the level of Bcl-2 had notably increased, and Bax had decreased after the LPS stimulus when the XIST gene was silenced (Fig. 2G). The activations of MAPK14 and its related molecules p-ERK1 and p-ERK2 by LPS stimulus apoptosis was assessed by flow cytometry. F, G The protein expressions were detected using Western blotting. H, I Expressions of XIST and miR-132-3p were measured by qRT-PCR. $* p<0.05, * * p<0.01$

were also remarkably diminished (Fig. 2H). The above results indicated that knockdown of XIST had a protective role in LPS-induced MEL-12 cells damage.

\section{MiR-132-3p expression was negatively regulated by XIST}

To elucidate the mechanism of XIST in ALI, the potential binding site between XIST and miR-132-3p was predicted (Fig. 3A). Dual-luciferase reporter assay showed a 
A

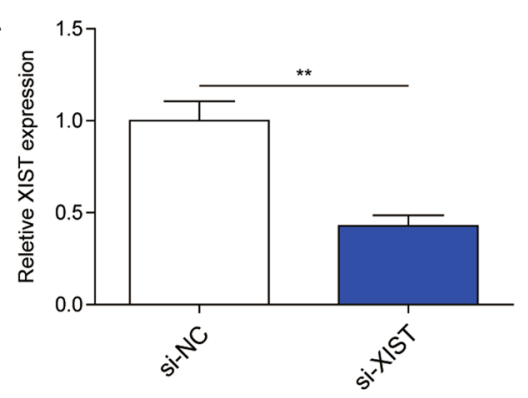

D

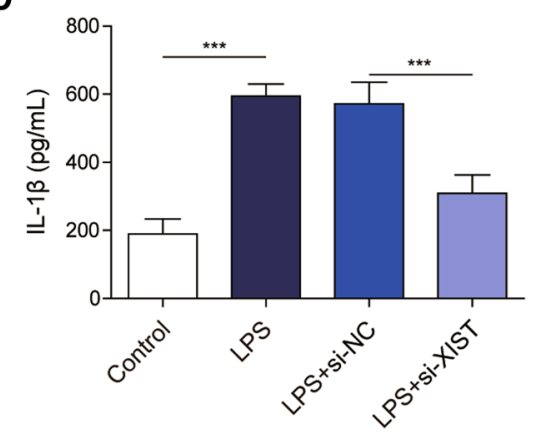

E
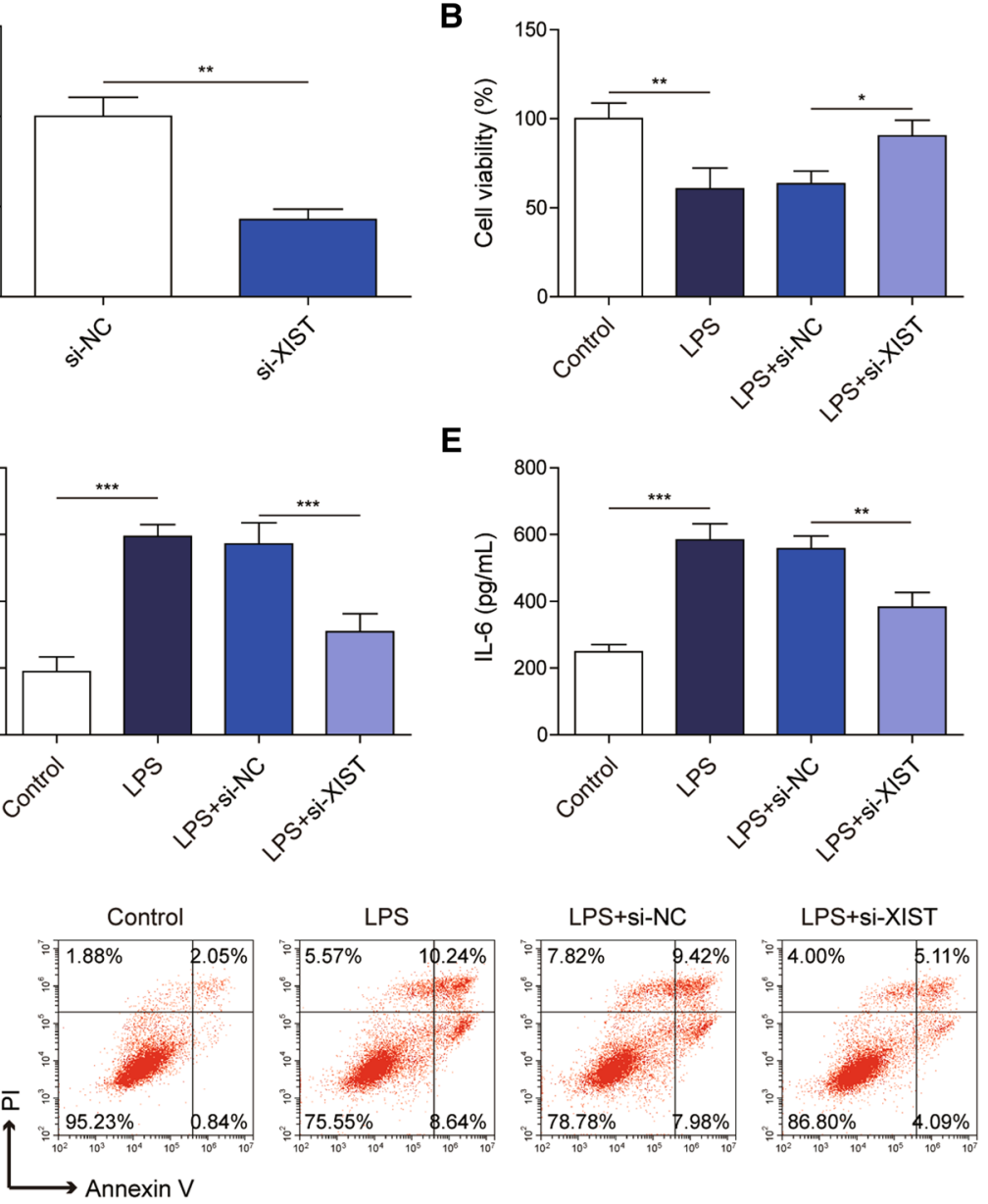

H
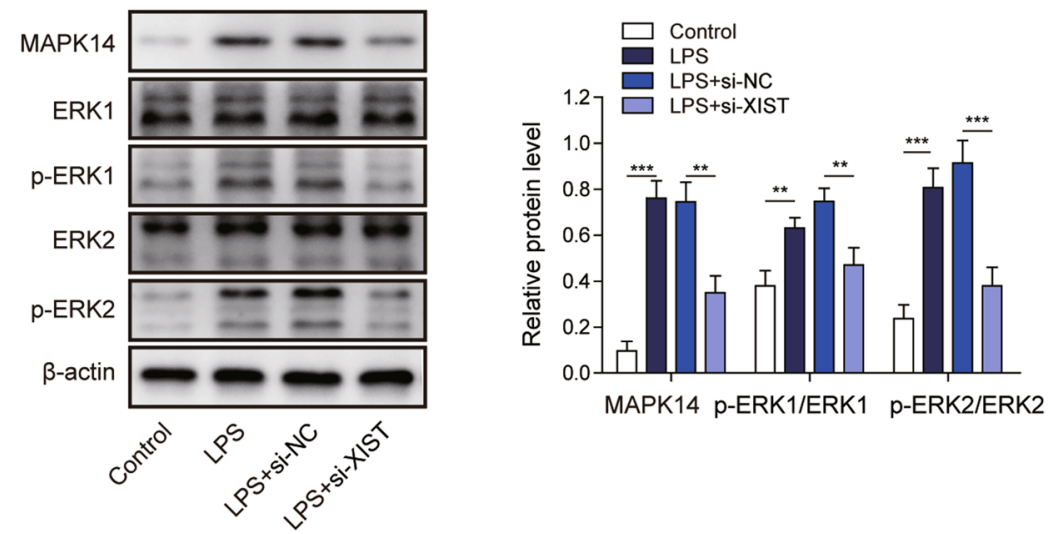

C

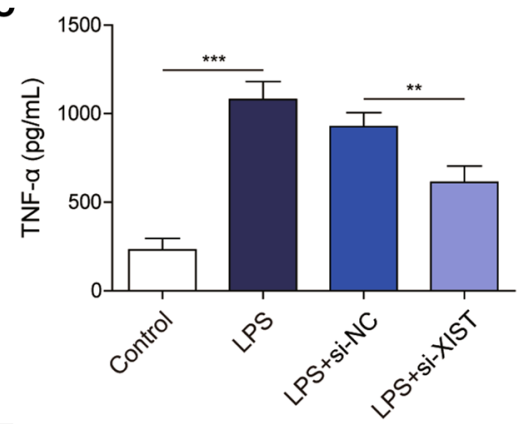

$\mathbf{F}$

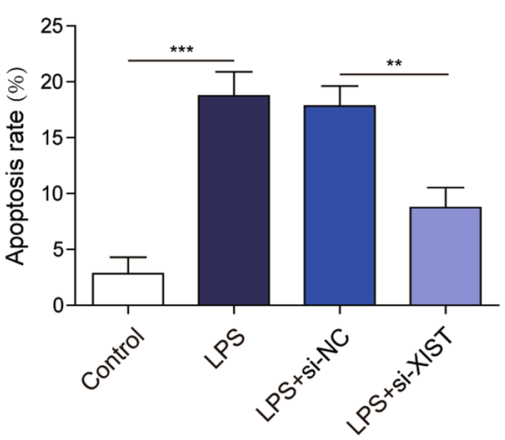

G
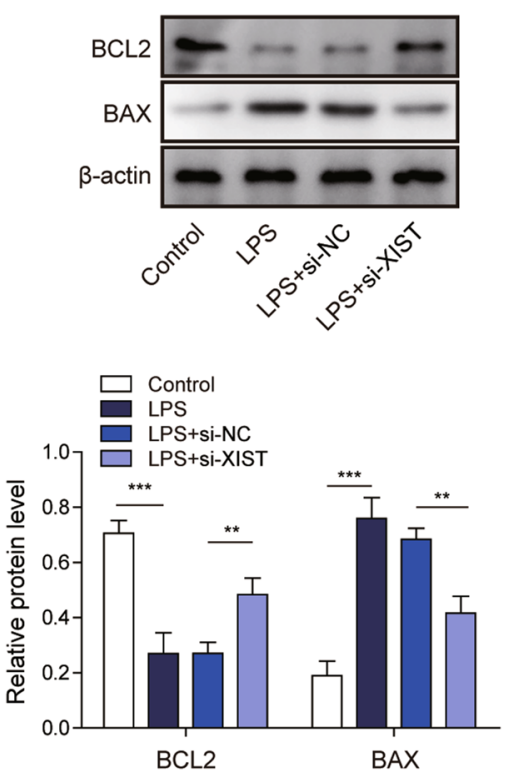

Fig. 2 XIST knockdown promoted cell viability and alleviated LPS-induced in MEL-12 cells damage. A Expression of XIST was checked after XIST knockdown by qRT-PCR. B Cell viabilities were detected in different groups by CCK-8 assay. C-E The concentra-

significant decline of luciferase activity was found when XIST-wt co-transfected with miR-132-3p than co-transfected with mimic NC, but there was no change between XISTmut co-transfected with mimic NC and XIST-mut co-transfected with miR-132-3p (Fig. 3B). Besides, the miR-132-3p tion of cytokines was measured in different groups by ELISA. F Cell apoptosis was assayed in different groups by flow cytometry. $\mathbf{G}, \mathbf{H}$ Protein levels of Bcl-2, Bax, MAPK14, ERK1/2, and p-ERK1/2 were detected by Western blotting. $* p<0.05, * * p<0.01$

expression level was upregulated after XIST knockdown (Fig. 3C). Further experiments indicated miR-132-3p level was significantly decreased when XIST was overexpressed (Fig. 3C). The above data suggested that XIST could directly target miR-132-3p in MEL-12 cells. 
A

$\begin{array}{rc}\text { XIST-wt } & \text { 5'-GCCGCAUUCUUUUGGACUGUUA-3' } \\ & |||\quad||||||||||| \\ \text { mmu-miR-132-3p } & \text { 3'-GCUGGUACCGACAUCUGACAAU-5' } \\ \text { XIST-mut } & \text { 5'-GCCGCAUUCUUUUGCUGACAAA-3' }\end{array}$

B

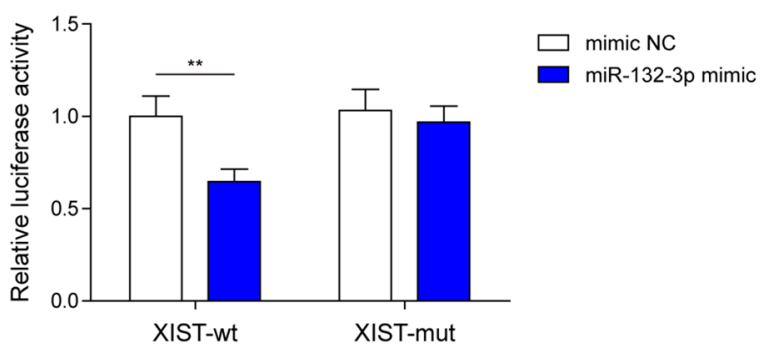

C

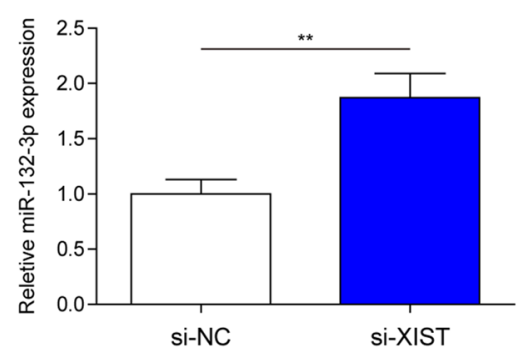

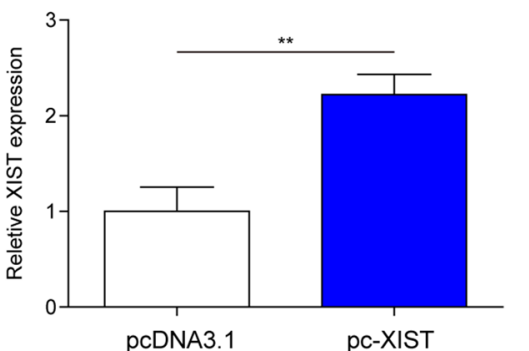

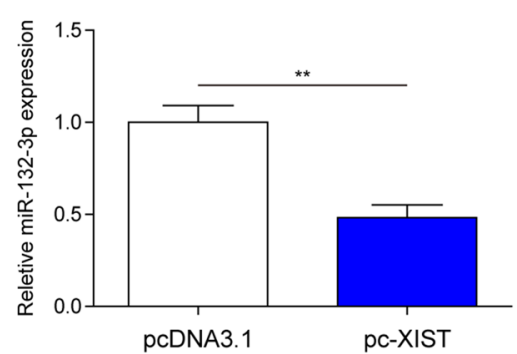

Fig. 3 XIST directly targeted on miR-132-3p. A The potential binding sites between XIST and miR-132-3p were predicted by StarBase. B Luciferase reporter assay was used to examine the relative luciferase activity of XIST-wt/mut after co-transfection with miR-132-3p mimic or mimic NC. C Expressions of miR-132-3p and XIST were detected after transfection with si-XIST/si-NC or transfection with pcDNA 3.1 and pc-XIST by qRT-PCR. ${ }^{*} p<0.05$; $* p<0.01$

\section{A}

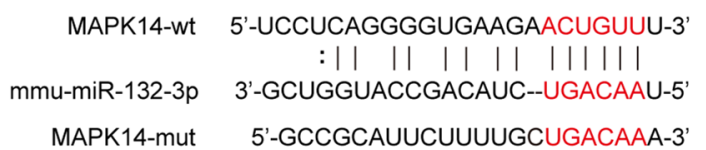

C

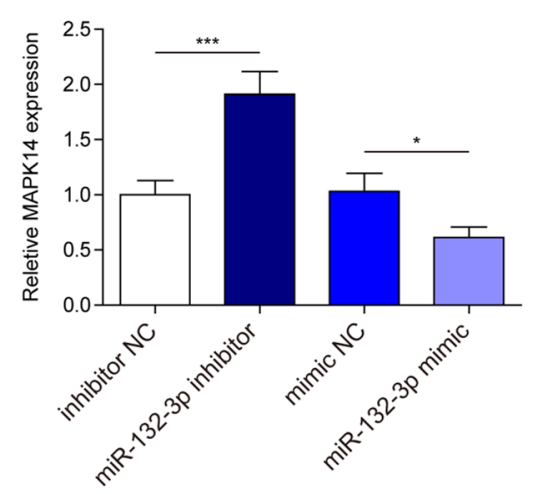

D

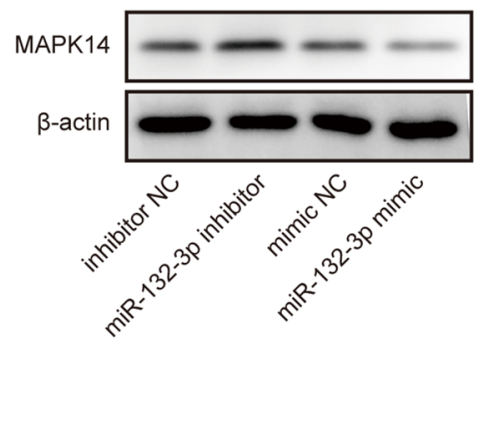

B

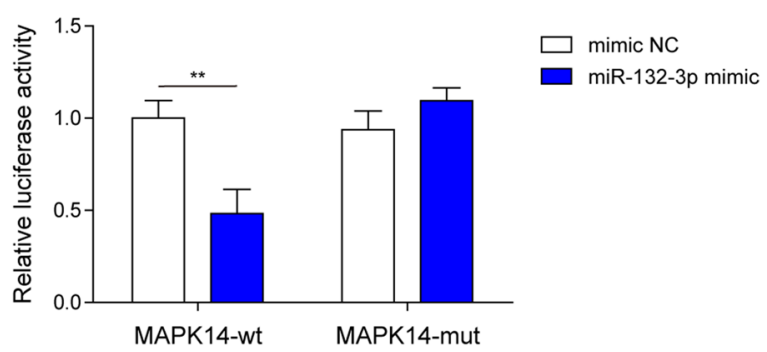

Fig. 4 MiR-132-3p directly targeted on MAPK14. A The potential binding sites of miR-132-3p and MAPK14 were predicted by StarBase. B Luciferase reporter assay was used to examine the relative luciferase activity after co-transfection of MAPK14-wt/mut luciferase vector with miR-132-3p mimic or mimic NC. C MAPK14 expres- sion was detected after transfection with miRNA inhibitor control and miR-132-3p inhibitor or mimic NC and miR-132-3p mimic. D Protein level of MAPK14 was detected after transfection with miRNA inhibitor control and miR-132-3p inhibitor or mimic NC and miR-132-3p mimic. ${ }^{*} p<0.05 ; * * p<0.01$ 
A

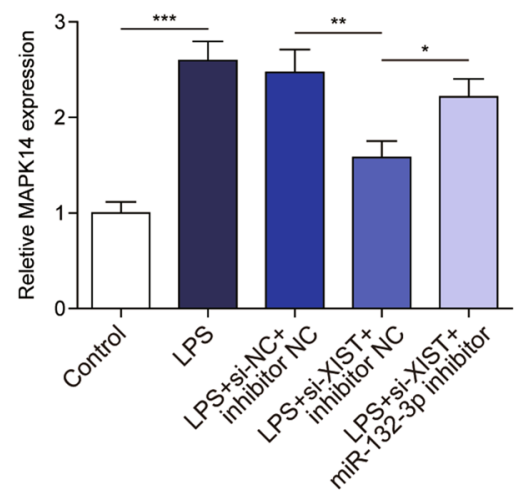

C

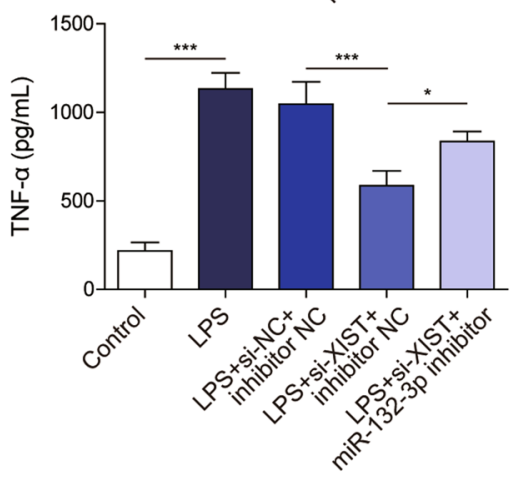

B

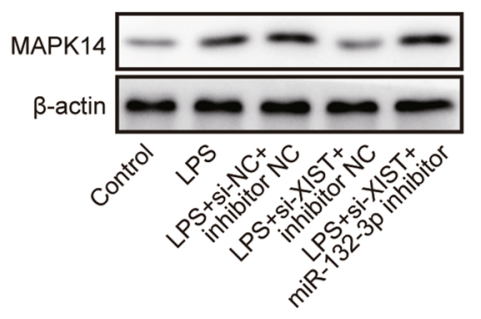

D

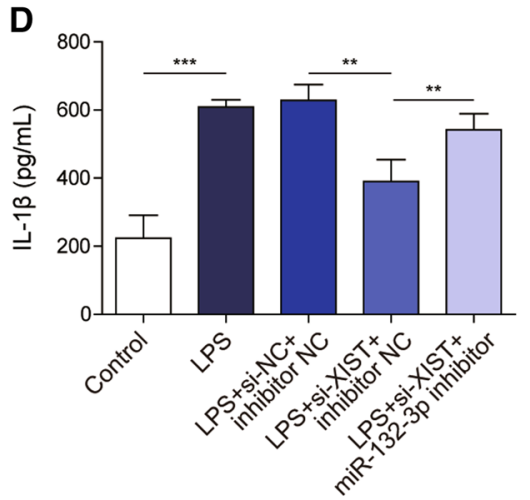

E
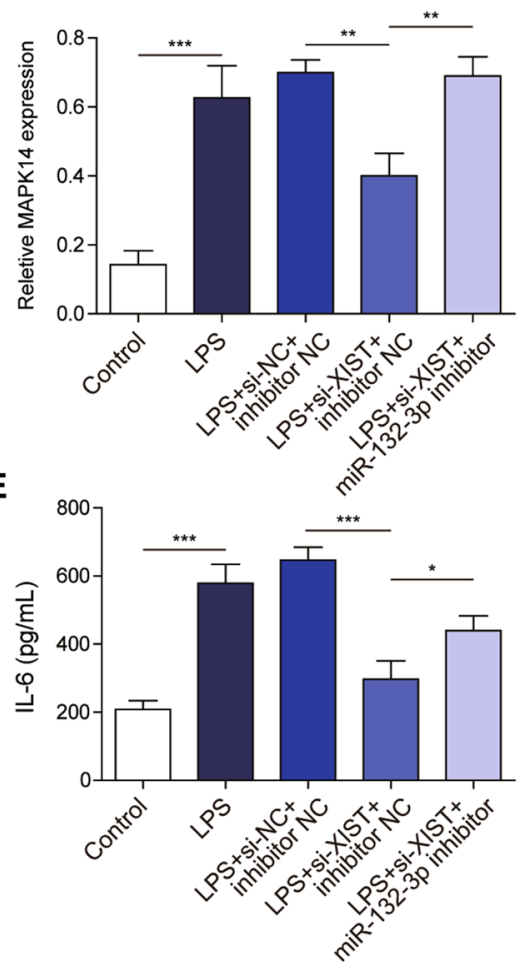

$\mathbf{F}$
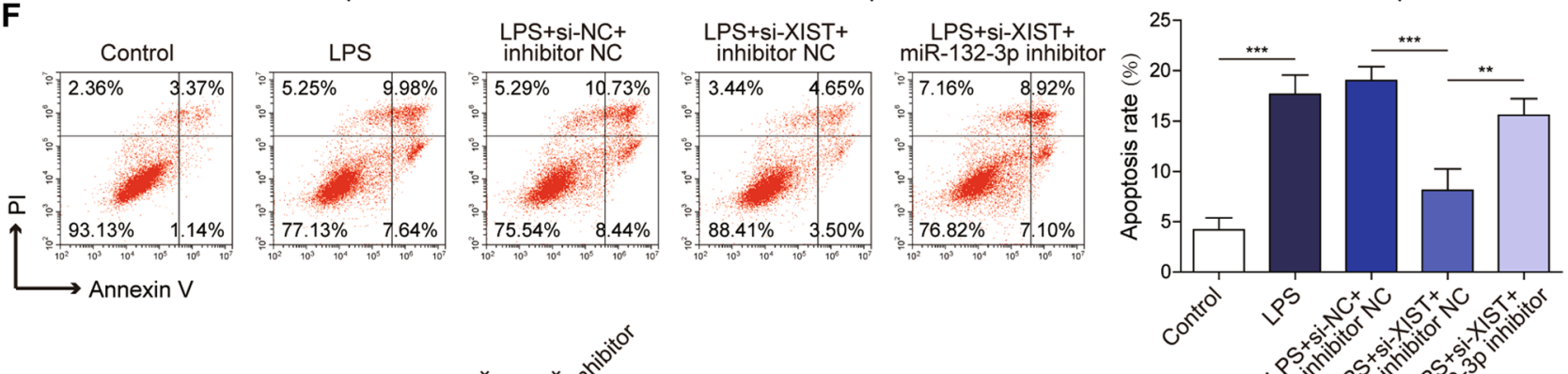

G

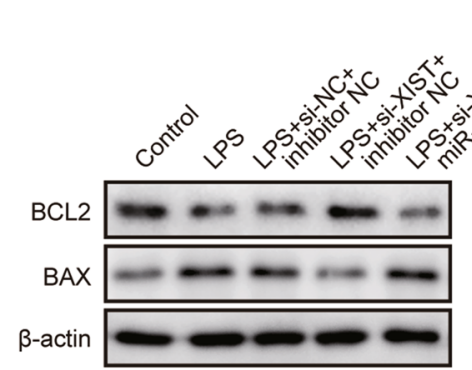

H
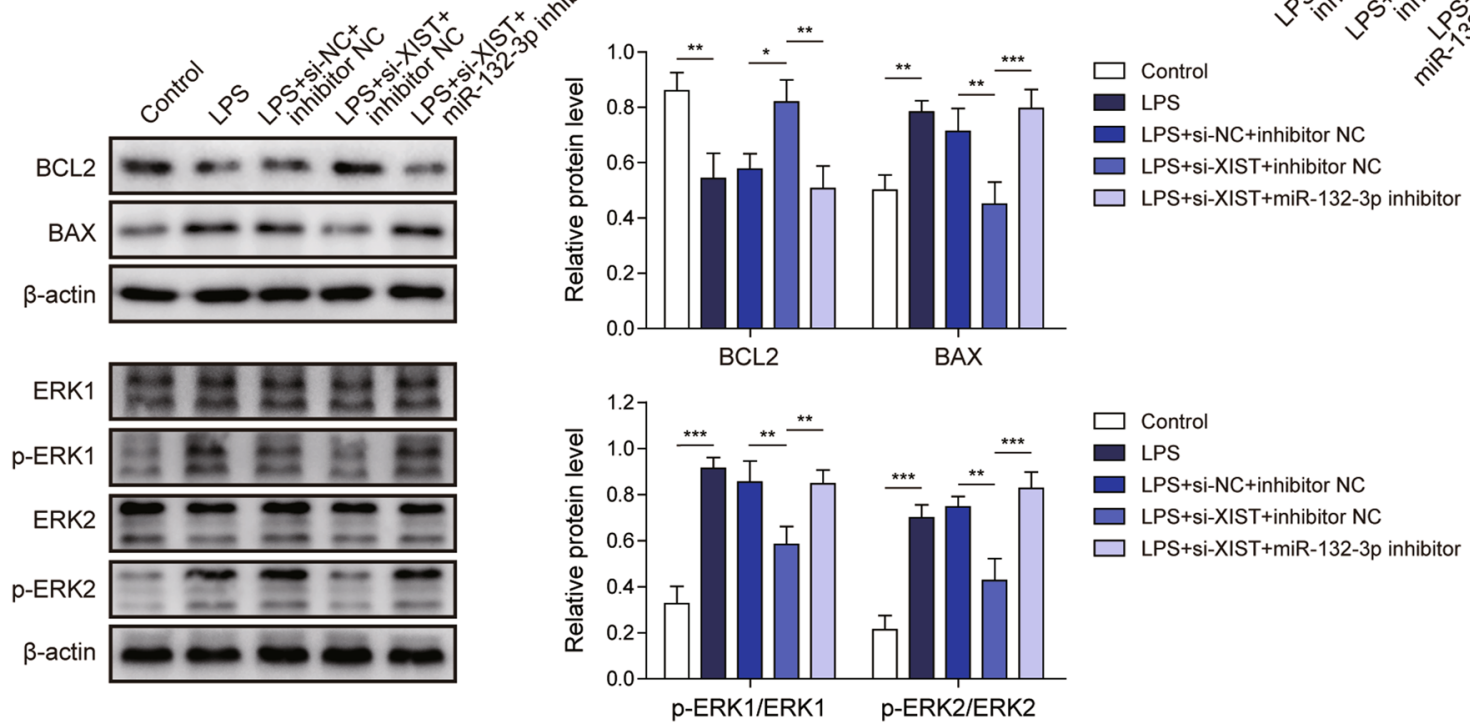
$\square$ Control
$\square$ LPS
PPS+si-NC+inhibitor NC
$\square$ LPS+si-XIST+inhibitor NC
$\square$ LPS+si-XIST+miR-132-3p inhibitor


४Fig. 5 XIST knockdown attenuated LPS-induced injury via miR132-3p/MAPK14 axis in MEL-12 cells. A MAPK14 expression was detected after transfection of si-NC or si-XIST together with miRNA inhibitor control or miR-132-3p inhibitor, followed by LPS treatment. B Protein level of MAPK14 was detected after transfection of si-NC or si-XIST together with miRNA inhibitor control or miR-132-3p inhibitor, followed by LPS treatment. C-E TNF- $\alpha$, IL-1 $\beta$, and IL-6 levels were detected after transfection of si-NC or si-XIST together with miRNA inhibitor control or miR-132-3p inhibitor, followed by LPS treatment. F Cell apoptosis was detected after transfection of si-NC or si-XIST together with miRNA inhibitor control or miR132-3p inhibitor, followed by LPS treatment. G, H Protein levels of Bcl-2, Bax, ERK1/2, and p-ERK1/2 were detected after transfection of si-NC or si-XIST with miRNA inhibitor control or miR-132-3p inhibitor, followed by LPS treatment. ${ }^{*} p<0.05 ; * * p<0.01$

\section{MiR-132-3p directly binds to 3'-UTR of MAPK14 to suppress its expression}

The target of miR-132-3p was predicted by the bioinformatics software of StarBase (Fig. 4A). We found that the luciferase activity in the MAPK14-wt 3'-UTR group was distinctively inhibited by miR-132-3p mimic (Fig. 4B). After transfection of miR-132-3p mimic, both MAPK14 mRNA (Fig. 4C) and protein levels (Fig. 4D) were significantly downregulated. In contrast, miR-132-3p inhibitor transfections showed the opposite effect on MAPK14 mRNA and protein expression (Fig. 4C and D). Based on the above results, we confirmed miR-132-3p directly targeted MAPK14 in MEL-12 cells.

\section{XIST knockdown attenuated LPS-induced injury via miR-132-3p/MAPK14 axis in MEL-12 cells}

We found there were potential interactions among XIST, miR-132-3p, and MAPK14. Therefore, MEL-12 cells were transfected with si-XIST or si-NC alone or together with miR-132-3p inhibitor or NC negative control and subsequently subjected to LPS for $24 \mathrm{~h}$. Then, MAPK14 levels were detected. As shown in Fig. 5A and B, MAPK14 was significantly downregulated for both mRNA and protein levels after XIST knockdown, and the miR-132-3p inhibitor partially antagonized this effect. This result indicated that XIST could regulate MAPK14 through miR-132-3p. Further experiments were carried out to investigate whether knockdown of XIST participated in the inflammatory response. The ELISA data showed that TNF- $\alpha$, IL- $1 \beta$, and IL-6 were obviously inhibited by XIST knockdown but partially reversed by a miR-132-3p inhibitor (Fig. 5C-E). Meanwhile, cell apoptosis was also decreased when XIST silencing but was counteracted by a miR-132-3p inhibitor (Fig. 5F). Besides, both Bax and p-ERK1/2 were dramatically downregulated, and the high expression of Bcl-2 by XIST knockdown was reversed by the miR-132-3p inhibitor (Fig. 5G and H). Totally, we found inhibition of XIST attenuated LPS-induced injuries by regulating the miR132-3p/MAPK14 axis.

\section{XIST knockdown mitigated LPS-induced ALI in mice through miR-132-3p/MAPK14 axis}

Based on the above data, we further wanted to explore whether knockdown of XIST could mitigate LPS-induced ALI in mice. After LPS stimulus in XIST knockdown mice, H\&E staining showed LPS treatment significantly increased the accumulation of inflammatory cells and abundant alveolar hemorrhage. XIST knockdown alleviated these pathological changes and decreased the score (Fig. 6A). Meanwhile, XIST knockdown distinctly reduced the lung wet/dry weight ratio, MDA, and cytokine secretions and increase the $\mathrm{PaO}_{2} /$ $\mathrm{FiO}_{2}$ in mice, indicating that inhibition of XIST alleviated LPS-induced ALI in mice (Fig. 6B-E). To confirm the levels of XIST in LPS-injured mice after delivering si-NC or si-XIST, we extracted RNAs from the isolated lung tissues and performed the qRT-PCR. Our results revealed that LPS stimulation could significantly increase the levels of XIST, but administration of si-XIST remarkably downregulated the XIST expression compared with that of the si-NC group. qRT-PCR (Fig. 6F) and western blot assays (Fig. 6G-H) also showed that inhibition of XIST upregulated miR-132-3p and diminished the activities of MAPK14, p-ERK1, and p-ERK2. Taken together, XIST knockdown protected LPSinduced ALI in vivo via miR-132-3p/MAPK14 axis.

\section{Discussion}

XIST and miR-132-3p can participate in inflammatory response through post-transcriptional control of gene expression. However, there are no reports about whether XIST and miR-132-3p participate in LPS-induced lung injury. In the present study, we confirmed that LPS-induced significant cell death and increased cytokine secretion. LPS stimuli also activated the MAPK signal and upregulated XIST level, but the miR-132-3p level was remarkably downregulated. All the data indicated that XIST, miR-132-3p, and MAPK14 were involved in LPS-induced ALI. Here, we first provided evidence that XIST knockdown prevented inflammatory response from LPS challenge by targeting the miR-132-3p/ MAPK14 pathway.

Neurotrophic recruitment to the lung and the alveolar and systematic release of pro-inflammatory cytokines are the main event responsible for the pulmonary inflammation in ALI and ARDS. A previous study showed ALI is typical in hospitalization pneumonia patients with positive microbiologic diagnosis [29]. Bacteria such as Pseudomonas aeruginosa and Acinetobacter baumannii were identified as 


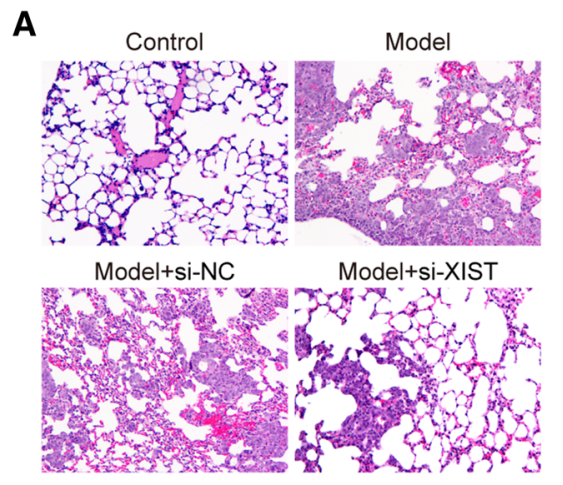

C
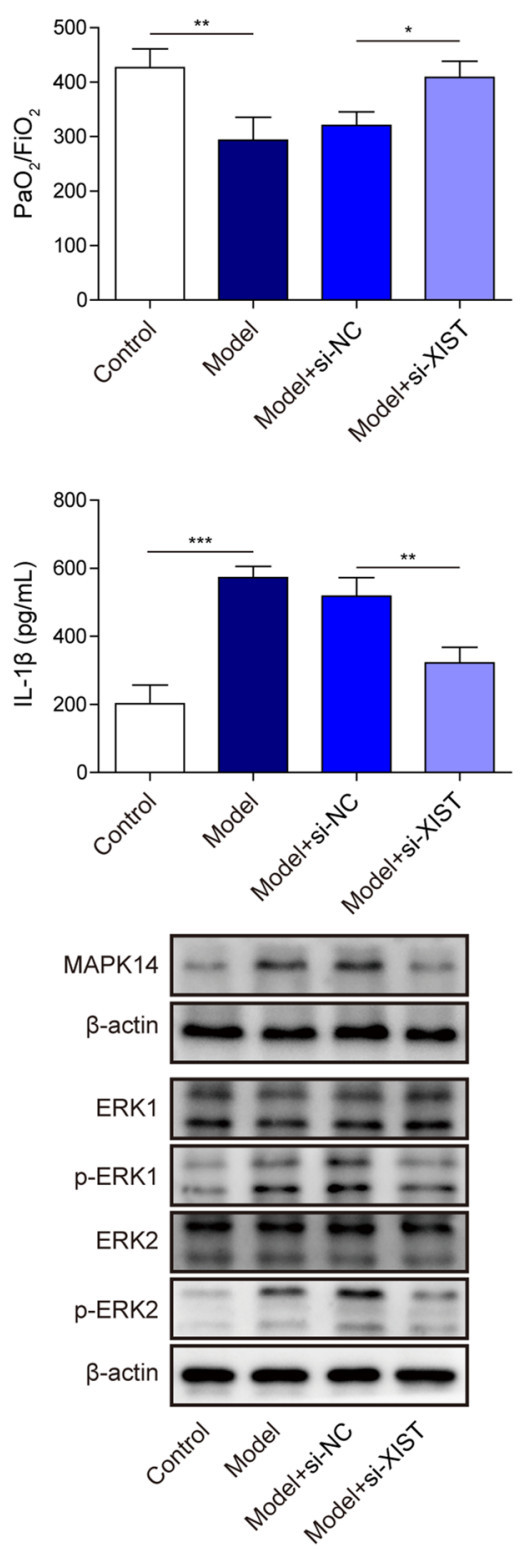

B

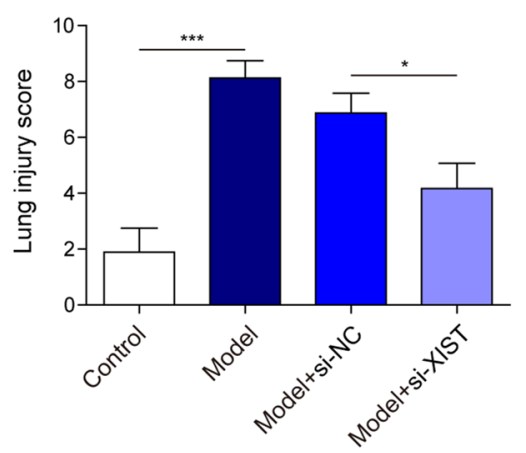

D

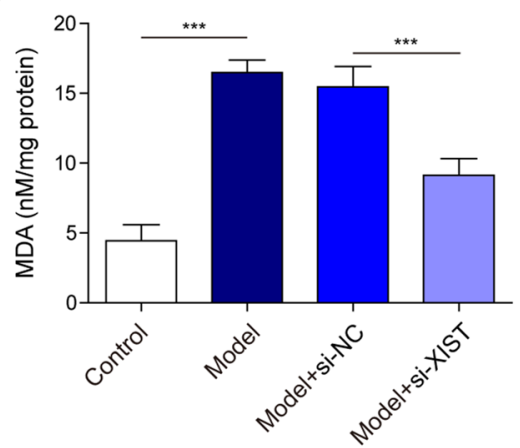

$\mathbf{F}$

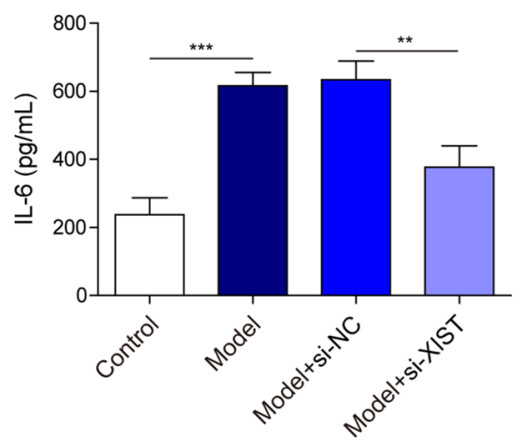

E
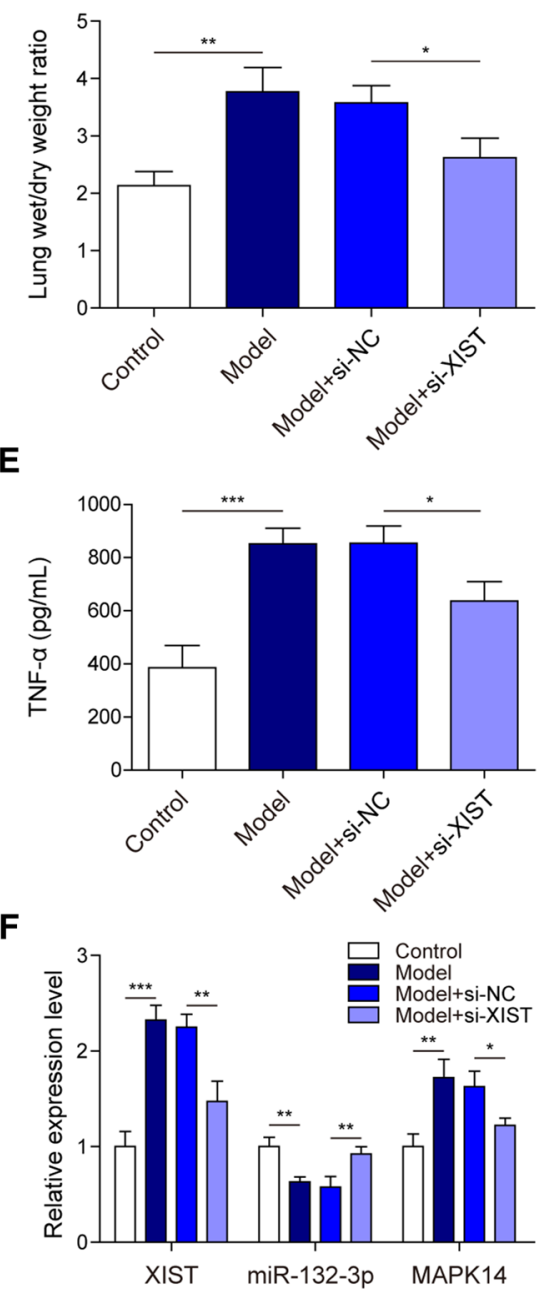

G
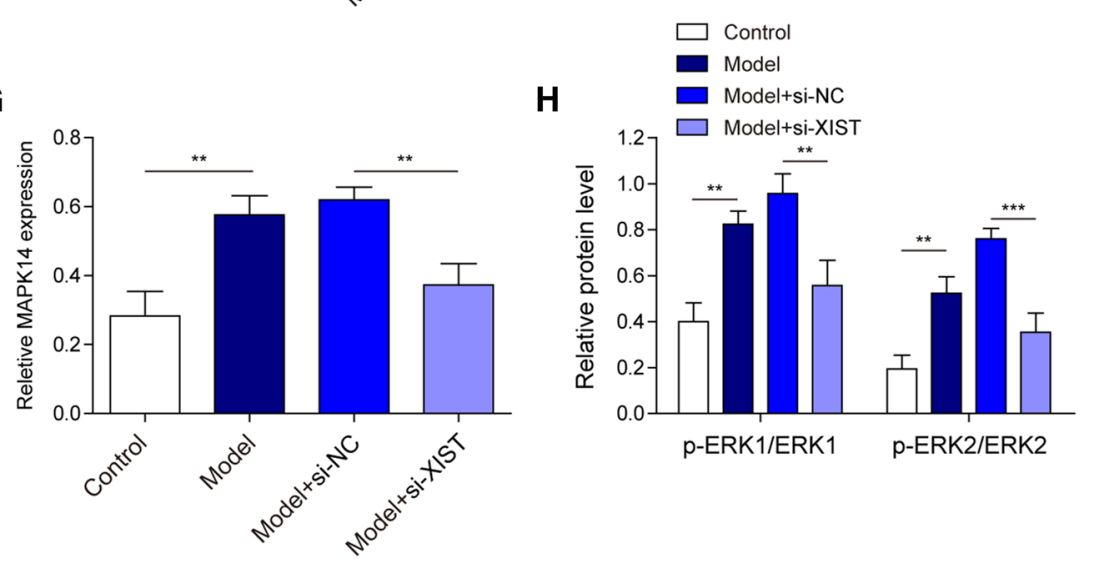

Fig. 6 XIST knockdown mitigated LPS-induced ALI in mice through miR-132-3p/MAPK14 axis. A The inflammatory conditions were assessed by H\&E staining in mice lung tissues. B Wet-to-dry weight ratio was measured in mice lung tissues. $\mathbf{C ~} \mathrm{PaO}_{2} / \mathrm{FiO}_{2}$ was assessed in mice lung tissue. D MDA contents were measured in mice lung tissue. $\mathbf{E}$ TNF- $\alpha$, IL- $1 \beta$, and IL-6 were analyzed in mice serum by ELISA. F XIST, miR-132-3p, and MAPK14 levels were detected in mice lung tissue by qRT-PCR. G, H Protein levels of MAPK14, ERK1/2, and p-ERK1/2 were detected in mice lung tissue. ${ }^{*} p<0.05$; $* * p<0.01$ 
significant pathogens, leading to pneumonia [29]. When LPS is released from those Gram-negative bacteria into the circulation, it can be recognized by inflammatory cells and subsequently triggers the pro-inflammatory response, which further causes multiple organ dysfunction. Although LPS was believed as an essential inducer for lung injury, the definite mechanisms remain unclear. Our experiments found that LPS significantly influenced cell viability, secretion of cytokines, and cell apoptosis. LPS stimuli also altered MAPK14, XIST, and miR-132-3p levels. All the data indicated that XIST, miR-132-3p, and MAPK14 were involved in LPS-induced ALI.

Previous studies pointed out that XIST was an oncogenic factor in multiple cancers $[14,19]$. Recently, several studies had revealed that LncRNAs were identified as a pivotal modulator in the inflammatory process [30, 31]. For example, LncRNA TUG1 could attenuate sepsis-induced acute inflammatory response and cell apoptosis via targeting miR33b-5p and GAB1 [30]. Knockdown of XIST had been identified as the primary modulator to alleviate the inflammatory response in spinal cord injury [32]. The MAPK pathway regulators include $\mathrm{p} 38$, JNK, and ERK1/2, which were considered a critical modulator of the inflammatory response in the lung [33]. Increased activity of MAPK, particularly p38 MAPK, could enhance the synthesis of inflammation mediators at the transcription and translation levels, which meant p38 MAPK might be a potential target to develop chemicals to inhibit inflammatory activity [34]. Recent studies showed that the suppression of the MAPK signaling pathway could attenuate LPS-induced pathological injuries and decreased expression of cytokines in ALI [35]. In our experiments, knockdown of XIST could increase cell viability, Bcl-2 and decrease the release of IL- $1 \beta$, IL- 6 and TNF- $\alpha$. Noticeably, knockdown of XIST inhibited the activation of MAPK14 as well as p-ERK1/2, which meant MAPK14 was involved in the inflammatory response regulated by XIST.

A series of studies had confirmed that miRNAs actively participated in the regulations of the pathogenesis of lung diseases such as pulmonary hypertension [36], lung cancer [37], pulmonary fibrosis [38], and chronic obstructive pulmonary disease [39]. Expression profiling assay of miRNAs in LPS-induced ALI suggested that the dysregulated miRNAs were essential for explaining the mechanism for ALI [40], but the definite mechanism is still under investigation. LncRNAs were identified to regulate the coding mRNAs that harbor the same miRNA target sequence by binding with the shared inhibitory miRNAs [41]. Hence, the crosstalk among LncRNAs, miRNAs might regulate the physiological and pathological processes through inhibiting targeting mRNA translation or promoting mRNA degradation [41]. Our data revealed that XIST silencing decreased cell apoptosis and secretions of pro-inflammatory cytokines. Additionally, knockdown of XIST enhanced Bcl-2 and inhibited
Bax and MAPK14 expressions. Totally, we evidenced that XIST knockdown suppressed inflammatory response in ALI.

Many studies have shown that XIST could interact with miRNAs [30, 31]. For example, XIST was reported to deteriorate cervical cancer through regulating miR-124, miR889-3p [42], suppress glioma progression by the upregulation of miR-204-5p [43], and accelerate the Primary graft dysfunction by binding to miR-21 [44]. However, the underlying molecular mechanism of XIST and miRNA in ALI has not been elucidated. We found that inhibition of XIST expression could directly upregulate miR-132-3p in MEL12. We believed that the interaction between XIST and miR$132-3 p$ had a regulatory role in ALI from the above results.

MAPK family includes ERK, c-Jun, and p38 MAPK subfamilies. After binding and activating cell surface receptors, LPS induces activation of MAP3K, MAP2K, and MAPK. Accumulated studies had confirmed that MAPK could be regulated by XIST [45] or miR-132 [46]. Using bioinformatics analysis, we found MAPK14 contained a potential binding site for miR-132-3p. So, we proposed that the cross interactions of XIST and miR-132-3p participated in the pathophysiological process of ALI via the MAPK14 signaling pathway. Here, we observed that miR-132-3p directly targeted MAPK14 to inhibit its expression. XIST knockdown significantly inactivated p-ERK1 and p-ERK2, which led to decreased cytokine releases and a protective role in cell survival, while miR-132-3p inhibition exerted a proinflammatory effect. In LPS-induced murine lung tissues, miR-132-3p was upregulated when XIST was inhibited, whereas MAPK14 expression was downregulated. So, miR132-3p was downregulated by XIST. In contrast, XIST was positively correlated with MAPK14.

In conclusion, this study firstly validated that XIST was significantly upregulated and miR-132-3p was markedly downregulated in LPS-induced MLE-12 cells and LPStreated mice. Downregulation of XIST alleviated LPSinduced apoptosis and inflammatory injuries in MLE-12 cells and mice through regulating the miR-132-3p/MAPK14 axis. This work may provide us a novel understanding of the function of XIST in the pathological progression of ALI and will provide us a new therapeutic strategy to cure bacterial infection-induced lung injury.

Acknowledgements We would like to give our sincere gratitude to the reviewers for their constructive comments.

Author contributions CL: Conceptualization, Writing- Original draft preparation, Validation, Visualization, and Methodology. J-HL: Methodology. JS: Software. W-JL: Data curation. J-QZ: Visualization. Z-HZ: Investigation. QW: Conceptualization, Writing-Original draft preparation, Supervision, and Writing-Reviewing and Editing.

Data availability All data collected and analyzed during the current study are available from the corresponding author on reasonable request. 


\section{Declarations}

Conflict of interest The authors declare that they have no competing interests to disclose.

Consent for publication All the authors approved the publication.

Ethical approval This study was approved by the Ethics Committee of the Tianjin Medical University, and all experiments were performed under accordance with the National Institutes of Health guidelines.

\section{References}

1. Matthay MA, Zemans RL, Zimmerman GA, Arabi YM, Beitler JR, Mercat A, Herridge M, Randolph AG, Calfee CS (2019) Acute respiratory distress syndrome. Nat Rev Dis Primers 5:18. https:// doi.org/10.1038/s41572-019-0069-0

2. Parekh D, Dancer RC, Thickett DR (2011) Acute lung injury. Clin Med (Lond) 11:615-618. https://doi.org/10.7861/clinmedicine. 11-6-615

3. Reutershan J, Basit A, Galkina EV, Ley K (2005) Sequential recruitment of neutrophils into lung and bronchoalveolar lavage fluid in LPS-induced acute lung injury. Am J Physiol Lung Cell Mol Physiol 289:L807-L815. https://doi.org/10.1152/ajplung. 00477.2004

4. Yao RW, Wang Y, Chen LL (2019) Cellular functions of long noncoding RNAs. Nat Cell Biol 21:542-551. https://doi.org/10. 1038/s41556-019-0311-8

5. Zampetaki A, Albrecht A, Steinhofel K (2018) Long non-coding RNA structure and function: is there a link? Front Physiol 9:1201. https://doi.org/10.3389/fphys.2018.01201

6. Sado T, Brockdorff N (2013) Advances in understanding chromosome silencing by the long non-coding RNA Xist. Philos Trans R Soc Lond B Biol Sci 368:20110325. https://doi.org/10.1098/rstb. 2011.0325

7. Pintacuda G, Young AN, Cerase A (2017) Function by structure: spotlights on Xist long non-coding RNA. Front Mol Biosci 4:90. https://doi.org/10.3389/fmolb.2017.00090

8. McHugh CA, Chen CK, Chow A, Surka CF, Tran C, McDonel P, Pandya-Jones A, Blanco M, Burghard C, Moradian A, Sweredoski MJ, Shishkin AA, Su J, Lander ES, Hess S, Plath K, Guttman M (2015) The Xist lncRNA interacts directly with SHARP to silence transcription through HDAC3. Nature 521:232-236. https://doi. org/10.1038/nature14443

9. Li C, Wan L, Liu Z, Xu G, Wang S, Su Z, Zhang Y, Zhang C, Liu X, Lei Z, Zhang HT (2018) Long non-coding RNA XIST promotes TGF-beta-induced epithelial-mesenchymal transition by regulating miR-367/141-ZEB2 axis in non-small-cell lung cancer. Cancer Lett 418:185-195. https://doi.org/10.1016/j.canlet.2018. 01.036

10. Wang H, Shen Q, Zhang X, Yang C, Cui S, Sun Y, Wang L, Fan $X, X u$ S (2017) The long non-coding RNA XIST controls nonsmall cell lung cancer proliferation and invasion by modulating miR-186-5p. Cell Physiol Biochem 41:2221-2229. https://doi.org/ $10.1159 / 000475637$

11. Sohrabifar N, Ghaderian SMH, Alipour Parsa S, Ghaedi H, Jafari $\mathrm{H}$ (2020) Variation in the expression level of MALAT1, MIAT and XIST lncRNAs in coronary artery disease patients with and without type 2 diabetes mellitus. Arch Physiol Biochem. https:// doi.org/10.1080/13813455.2020.1768410
12. Sharma A, Kaut O, Pavlova A, Frohlich H, Ahmad A, Schmitt I, El-Maarri O, Oldenburg J, Wullner U (2017) Skewed X-chromosome inactivation and XIST locus methylation levels do not contribute to the lower prevalence of Parkinson's disease in females. Neurobiol Aging 57:248-248. https://doi.org/10.1016/j.neurobiola ging.2017.05.021

13. Yue D, Guanqun G, Jingxin L, Sen S, Shuang L, Yan S, Minxue Z, Ping Y, Chong L, Zhuobo Z, Yafen W (2020) Silencing of long noncoding RNA XIST attenuated Alzheimer's disease-related BACE1 alteration through miR-124. Cell Biol Int 44:630-636. https://doi.org/10.1002/cbin.11263

14. Parodi S (2020) Xist noncoding RNA could act as a tumor suppressor gene in patients with classical Hodgkin's disease. J Cancer Res Ther 16:7-12. https://doi.org/10.4103/jcrt.JCRT_1055_16

15. Zhang Y, Zhu Y, Gao G, Zhou Z (2019) Knockdown XIST alleviates LPS-induced WI-38 cell apoptosis and inflammation injury via targeting miR-370-3p/TLR4 in acute pneumonia. Cell Biochem Funct 37:348-358. https://doi.org/10.1002/cbf.3392

16. Tang B, Li W, Ji T, Li X, Qu X, Feng L, Zhu Y, Qi Y, Zhu C, Bai $S$ (2020) Downregulation of XIST ameliorates acute kidney injury by sponging miR-142-5p and targeting PDCD4. J Cell Physiol 235:8852-8863. https://doi.org/10.1002/jcp.29729

17. Li Z, Zhang Y, Ding N, Zhao Y, Ye Z, Shen L, Yi H, Zhu Y (2019) Inhibition of lncRNA XIST improves myocardial I/R injury by targeting miR-133a through inhibition of autophagy and regulation of SOCS2. Mol Ther Nucleic Acids 18:764-773. https://doi. org/10.1016/j.omtn.2019.10.004

18. Shen C, Li J (2021) LncRNA XIST silencing protects against sepsis-induced acute liver injury via inhibition of BRD4 expression. Inflammation 44:194-205. https://doi.org/10.1007/ s10753-020-01321-X

19. Zhang M, Li Y, Wang H, Yu W, Lin S, Guo J (2019) LncRNA SNHG5 affects cell proliferation, metastasis and migration of colorectal cancer through regulating miR-132-3p/CREB5. Cancer Biol Ther 20:524-536. https://doi.org/10.1080/15384047.2018. 1537579

20. Su Q, Liu Y, Lv XW, Dai RX, Yang XH, Kong BH (2020) LncRNA TUG1 mediates ischemic myocardial injury by targeting miR-132-3p/HDAC3 axis. Am J Physiol Heart Circ Physiol 318:H332-H344. https://doi.org/10.1152/ajpheart.00444.2019

21. Pan Q, Kuang X, Cai S, Wang X, Du D, Wang J, Wang Y, Chen Y, Bihl J, Chen Y, Zhao B, Ma X (2020) miR-132-3p priming enhances the effects of mesenchymal stromal cell-derived exosomes on ameliorating brain ischemic injury. Stem Cell Res Ther 11:260. https://doi.org/10.1186/s13287-020-01761-0

22. Xie W, Lu Q, Wang K, Lu J, Gu X, Zhu D, Liu F, Guo Z (2018) miR-34b-5p inhibition attenuates lung inflammation and apoptosis in an LPS-induced acute lung injury mouse model by targeting progranulin. J Cell Physiol 233:6615-6631. https://doi.org/10. $1002 /$ jcp. 26274

23. Li H, Hou H, Liu S, Feng Y, Zhong W, Hu X, Yan N (2019) miR33 and RIP140 participate in LPS-induced acute lung injury. Turk J Med Sci 49:422-428. https://doi.org/10.3906/sag-1804-173

24. Neudecker V, Brodsky KS, Clambey ET, Schmidt EP, Packard TA, Davenport B, Standiford TJ, Weng T, Fletcher AA, Barthel L, Masterson JC, Furuta GT, Cai C, Blackburn MR, Ginde AA, Graner MW, Janssen WJ, Zemans RL, Evans CM, Burnham EL, Homann D, Moss M, Kreth S, Zacharowski K, Henson PM, Eltzschig HK (2017) Neutrophil transfer of miR-223 to lung epithelial cells dampens acute lung injury in mice. Sci Transl Med. https://doi.org/10.1126/scitranslmed.aah5360

25. Ding L, Gao X, Yu S, Sheng L (2020) miR-128-3p enhances the protective effect of dexmedetomidine on acute lung injury in septic mice by targeted inhibition of MAPK14. J Bioenerg Biomembr 52:237-245. https://doi.org/10.1007/s10863-020-09842-8 
26. Hanieh H, Alzahrani A (2013) MicroRNA-132 suppresses autoimmune encephalomyelitis by inducing cholinergic anti-inflammation: a new Ahr-based exploration. Eur J Immunol 43:2771-2782. https://doi.org/10.1002/eji.201343486

27. Roux PP, Blenis J (2004) ERK and p38 MAPK-activated protein kinases: a family of protein kinases with diverse biological functions. Microbiol Mol Biol Rev 68:320-344. https://doi.org/10. 1128/MMBR.68.2.320-344.2004

28. Lv H, Liu Q, Wen Z, Feng H, Deng X, Ci X (2017) Xanthohumol ameliorates lipopolysaccharide (LPS)-induced acute lung injury via induction of AMPK/GSK3beta-Nrf2 signal axis. Redox Biol 12:311-324. https://doi.org/10.1016/j.redox.2017.03.001

29. Kojicic M, Li G, Hanson AC, Lee KM, Thakur L, Vedre J, Ahmed A, Baddour LM, Ryu JH, Gajic O (2012) Risk factors for the development of acute lung injury in patients with infectious pneumonia. Crit Care 16:R46. https://doi.org/10.1186/cc11247

30. Qiu N, Xu X, He Y (2020) LncRNA TUG1 alleviates sepsisinduced acute lung injury by targeting miR-34b-5p/GAB1. BMC Pulm Med 20:49. https://doi.org/10.1186/s12890-020-1084-3

31. Wang S, Cao F, Gu X, Chen J, Xu R, Huang Y, Ying L (2019) LncRNA XIST, as a ceRNA of miR-204, aggravates lipopolysaccharide-induced acute respiratory distress syndrome in mice by upregulating IRF2. Int J Clin Exp Pathol 12:2425-2434

32. Zhao Q, Lu F, Su Q, Liu Z, Xia X, Yan Z, Zhou F, Qin R (2020) Knockdown of long noncoding RNA XIST mitigates the apoptosis and inflammatory injury of microglia cells after spinal cord injury through miR-27a/Smurf1 axis. Neurosci Lett 715:134649. https:// doi.org/10.1016/j.neulet.2019.134649

33. Li D, Ren W, Jiang Z, Zhu L (2018) Regulation of the NLRP3 inflammasome and macrophage pyroptosis by the p38 MAPK signaling pathway in a mouse model of acute lung injury. Mol Med Rep 18:4399-4409. https://doi.org/10.3892/mmr.2018.9427

34. El-Gamal MI, Anbar HS, Tarazi H, Oh CH (2019) Discovery of a potent p38alpha/MAPK14 kinase inhibitor: synthesis, in vitro/in vivo biological evaluation, and docking studies. Eur J Med Chem 183:111684. https://doi.org/10.1016/j.ejmech.2019.111684

35. Nie Y, Wang Z, Chai G, Xiong Y, Li B, Zhang H, Xin R, Qian X, Tang Z, Wu J, Zhao P (2019) Dehydrocostus lactone suppresses LPS-induced acute lung injury and macrophage activation through NF-kappaB signaling pathway mediated by p38 MAPK and Akt. Molecules. https://doi.org/10.3390/molecules24081510

36. Herrera-Rivero M, Zhang R, Heilmann-Heimbach S, Mueller A, Bagci S, Dresbach T, Schroder L, Holdenrieder S, Reutter HM, Kipfmueller F (2018) Circulating microRNAs are associated with pulmonary hypertension and development of chronic lung disease in congenital diaphragmatic hernia. Sci Rep 8:10735. https://doi. org/10.1038/s41598-018-29153-8
37. Lelli D, Pedone C, Majeed M, Sahebkar A (2017) Curcumin and lung cancer: the role of microRNAs. Curr Pharm Des 23:34403444. https://doi.org/10.2174/1381612823666170109144818

38. Rajasekaran S, Rajaguru P, Sudhakar Gandhi PS (2015) MicroRNAs as potential targets for progressive pulmonary fibrosis. Front Pharmacol 6:254. https://doi.org/10.3389/fphar.2015.00254

39. Huang X, Zhu Z, Guo X, Kong X (2019) The roles of microRNAs in the pathogenesis of chronic obstructive pulmonary disease. Int Immunopharmacol 67:335-347. https://doi.org/10.1016/j.intimp. 2018.12.013

40. Cao Y, Lyu YI, Tang J, Li Y (2016) MicroRNAs: Novel regulatory molecules in acute lung injury/acute respiratory distress syndrome. Biomed Rep 4:523-527. https://doi.org/10.3892/br. 2016.620

41. Marshall EA, Stewart GL, Sage AP, Lam WL, Brown CJ (2019) Beyond sequence homology: cellular biology limits the potential of XIST to act as a miRNA sponge. PLoS ONE 14:e221371. https://doi.org/10.1371/journal.pone.0221371

42. Liu X, Xie S, Zhang J, Kang Y (2020) Long noncoding RNA XIST contributes to cervical cancer development through targeting miR-889-3p/SIX1 axis. Cancer Biother Radiopharm. https:// doi.org/10.1089/cbr.2019.3318

43. Shen J, Xiong J, Shao X, Cheng H, Fang X, Sun Y, Di G, Mao J, Jiang X (2020) Knockdown of the long noncoding RNA XIST suppresses glioma progression by upregulating miR-204-5p. J Cancer 11:4550-4559. https://doi.org/10.7150/jca.45676

44. Li J, Wei L, Han Z, Chen Z, Zhang Q (2020) Long non-coding RNA X-inactive specific transcript silencing ameliorates primary graft dysfunction following lung transplantation through microRNA-21-dependent mechanism. EBioMedicine 52:102600. https://doi.org/10.1016/j.ebiom.2019.102600

45. Li L, Lv G, Wang B, Kuang L (2018) The role of lncRNA XIST/ miR-211 axis in modulating the proliferation and apoptosis of osteoarthritis chondrocytes through CXCR4 and MAPK signaling. Biochem Biophys Res Commun 503:2555-2562. https://doi.org/ 10.1016/j.bbrc.2018.07.015

46. Lei Z, van Mil A, Brandt MM, Grundmann S, Hoefer I, Smits M, El Azzouzi H, Fukao T, Cheng C, Doevendans PA, Sluijter JP (2015) MicroRNA-132/212 family enhances arteriogenesis after hindlimb ischaemia through modulation of the Ras-MAPK pathway. J Cell Mol Med 19:1994-2005. https://doi.org/10.1111/ jcmm. 12586

Publisher's Note Springer Nature remains neutral with regard to jurisdictional claims in published maps and institutional affiliations. 